\title{
COVID-19s Impact on the Hispanic Community: How Understanding Culture Can Improve Outcomes
}

\author{
Yamilet Guerra ${ }^{1}$ and Brenda Hudson, MA ${ }^{1,2}$ \\ ${ }^{1}$ Indiana University School of Medicine, ${ }^{2}$ Indiana Clinical \& Translational Sciences Institute
}

\section{Background}

A disproportionate burden of SARS-Cov-2 infection, or coronavirus disease 2019 (Covid-19), and death are highest among racial and ethnic minority groups. Based on data available on June 12, 2020, Hispanic people are more likely to acquire COVID-19 and have higher incidence of hospitalization and death compared to their white, non-Hispanic counterparts. While this issue is complex, many have hypothesized that the difference is due to societal factors and communication methods. The aim of this project was to evaluate information related to how the Hispanic population is affected by COVID-19 and how communications about the disease should be designed based on past research, physician input, and cultural sensitivities.

\section{Methods}

We conducted a thorough literature search on COVID-19 articles, both peer reviewed and grey literature, evaluating race and ethnic differences in disease prevalence and severity.

Additionally, we conducted interviews with a small number of Indiana doctors who treat Hispanic patients to obtain a doctor's perspective on the Hispanic community's needs during the pandemic and ways to help reduce prevalence.

\section{Results}

Physicians in Indiana believe the main approach to help the Hispanic community is by utilizing trusted community resources to communicate information and build relationships with patients over time. It is recommended to develop new methods to deliver essential information about COVID-19 through multiple mediums, in a clear way, and in Spanish with focus on the collective good of the family. In addition, it is important not to just translate resources from English to Spanish but to design materials addressing barriers specific to the Hispanic community.

\section{Conclusion}

More culturally tailored information should be released to educate the Hispanic community about COVID-19. This information will assist in the design of materials and initiatives for the Hispanic community that we hope will improve methods of communication and care delivery related to COVID-19. 
